\title{
WALSH-FOURIER COEFFICIENTS AND LOCALLY CONSTANT FUNCTIONS
}

\author{
WILLIAM R. WADE
}

\begin{abstract}
A condition on the Walsh-Fourier coefficients of a continuous function $f$ sufficient to conclude that $f$ is locally constant is obtained. The condition contains certain conditions identified earlier by Bockarev, Coury, Skvorcov and Wade, and Powell and Wade.
\end{abstract}

1. Introduction. Let $w_{0}, w_{1}, \ldots$ represent the Walsh functions, i.e., $w_{0} \equiv 1$ on the interval $[0,1]$, and $w_{n}$ (for $n>0$ ) is defined by

$$
w_{n}(x)=\prod_{j=0}^{\infty}(-1)^{x, n_{j}}, \quad x \in[0,1],
$$

where the exponents $x_{j}, n_{j}$ equal 0 or 1 and come from the binary expansions

$$
x=\sum_{j=0}^{\infty} x_{j} 2^{-j-1}, \quad n=\sum_{j=0}^{\infty} n_{j} 2^{j},
$$

and the finite expansion is used for dyadic rational $x \in[0,1)$.

Let $a_{0}(f), a_{1}(f), \ldots$ denote the Walsh-Fourier coefficients of an $f \in L^{1}[0,1]$ and recall that unlike the trigonometric case, there are restrictions on the rapidity with which $a_{k}(f)$ can decay for nonconstant, smooth $f$. For example, Bočkarev [1] proved that a continuous $f$ which satisfies

$$
\left|a_{k}(f)\right| \leqslant d_{k} \downarrow 0, \text { as } k \rightarrow \infty, \text { where } \sum d_{k}<\infty
$$

is constant on $[0,1]$. It follows that no nonconstant, continuous $f$ satisfies

$$
a_{k}(f)=O\left(\frac{1}{k(\log k)^{p}}\right), \quad \text { as } k \rightarrow \infty,
$$

for some $p>1$. He also constructed a nonconstant, continuous $f$ which satisfies (3) for $p=1$. Thus the growth of $a_{k}(f)$ for continuous $f$ is completely determined. The only drawback to Bočkarev's approach is that $\Sigma\left|a_{k}(f)\right|$ converges when (2) holds. Hence Bočkarev's theorem carries the tacit assumption that the Walsh-Fourier series of $f$ converges absolutely.

Received by the editors June 10, 1982.

1980 Mathematics Subject Classification. Primary $42 \mathrm{C} 10$.

Kev words and phrases. Walsh functions, Walsh-Dirichlet kernel, dyadic addition, Dini derivates. 
Coury [2] corrected this theoretical deficiency. He proved that a continuous $f$ which satisfies

$$
\lim _{p \rightarrow \infty} 2^{p} \sum_{m=p}^{\infty} \sum_{k=2^{m}}^{2^{m+1}-2}\left|a_{k}(f)-a_{k+1}(f)\right|=0
$$

is constant on $[0,1]$, and he provided examples to show that (4) applies in situations where $\Sigma\left|a_{k}(f)\right|=+\infty$. He also verified that condition (4) is implied by $\Sigma\left|a_{k}(f)\right|$ $<\infty$ when the coefficients $a_{k}(f)$ are eventually monotone decreasing in dyadic blocks, i.e.,

$$
a_{2^{m}}(f) \geqslant a_{2^{m+1}}(f) \geqslant \cdots \geqslant a_{2^{m+1}-1}(f)
$$

for $m$ sufficiently large. Thus Coury's results contain Bočkarev's theorem for continuous $f$ whose Walsh-Fourier coefficients are monotone decreasing.

In this paper conditions on the coefficients $a_{k}(f)$ sufficient to conclude that a continuous $f$ is locally constant are identified. When applied to the interval $[0,1]$, these conditions contain Coury's results cited above. In fact, it is shown that condition (4) can be relaxed by allowing certain gaps in the second sum. The technique used is simple and straightforward. We compute the Dini derivates of $f$.

2. Statement of results. For each integer $p \geqslant 0$ let $A(p, p)$ denote the collection of integers $k$ which satisfy $2^{p} \leqslant k<2^{p+1}$. For any pair of integers $m>p \geqslant 0$, set $A(m, p)=\left\{k: k\right.$ is an integer which satisfies $2^{m}+(2 l-1) 2^{p} \leqslant k<2^{m}+2 l 2^{p}$ for some $\left.l=1,2, \ldots, 2^{m-p-1}\right\}$. In $\S 3$ the following result is proved.

THEOREM 1. Let $Z$ be a subset of an interval $[a, b]$ and suppose that $Z$ is at most countable. If $f$ is continuous on $[a, b]$, and if

$$
\lim _{p \rightarrow \infty} 2^{p} \sum_{m=p}^{\infty} \sum_{k \in A(m, p)} a_{k}(f) w_{k}(x)
$$

exists and is nonnegative (respectively, nonpositive) for every $x \in[a, b] \sim Z$, then $f$ is constant on $[a, b]$.

Let $B(p, p)=A(p, p) \sim\left\{2^{p+1}-1\right\}$ and set

$$
B(m, p)=A(m, p) \sim\left\{2^{m}+2 l 2^{p}-1: l=1,2, \ldots, 2^{m-p-1}\right\}
$$

for $m>p, p=0,1, \ldots$ Notice that $B(m, p)$ contains about half the integers in $\left[2^{m}, 2^{m+1}-2\right]$ for $m=p, p+1, \ldots$ Thus the following result, whose proof is given in $\S 4$, contains Coury's theorem cited above.

THEOREM 2. If $f$ is continuous on $[0,1]$ and if the Walsh-Fourier coefficients of $f$ satisfy

$$
\lim _{p \rightarrow \infty} 2^{p} \sum_{m=p}^{\infty} \sum_{k \in B(m, p)}\left|a_{k}(f)-a_{k+1}(f)\right|=0,
$$

then $f$ is constant. 
In particular, no nonconstant, continuous $f$ has coefficients $a_{k} \equiv a_{k}(f)$ which are eventually monotone decreasing in dyadic blocks and satisfy

$$
\lim _{p \rightarrow \infty} 2^{p} \sum_{m=p}^{\infty} \sum_{l=1}^{2^{m}}\left(a_{2^{m}+(2 l-1) 2^{p}}-a_{2^{m}+2 / 2^{p}}\right)=0 .
$$

This corollary generalizes Theorem 2 in [2].

Notice that condition (7) surely holds if

$$
\lim _{p \rightarrow \infty} 2^{p} \sum_{m=p}^{\infty} \sum_{k \in B(m, p)}\left|a_{k}(f)\right|=0 .
$$

Thus the monotone condition in (2) can be dropped if the condition $\Sigma\left|a_{k}(f)\right|<\infty$ is strengthened to (9). Also notice that $k \in B(m, p)$ implies that $2^{p}<k$. Hence (9) holds if $\sum k\left|a_{k}(f)\right|<\infty$ (see Corollary 6 in [4]).

3. Estimating Dini derivates. We shall prove the following result in this section.

THEOREM 3. Let $x \in[0,1]$ be a dyadic irrational and suppose that

$$
f(t)=\lim _{n \rightarrow \infty} \sum_{k=0}^{2^{n}-1} a_{k} w_{k}(t)
$$

exists and is finite in a neighborhood of $x$. If

$$
\lim _{p \rightarrow \infty} 2^{p} \sum_{m=p}^{\infty} \sum_{k \in A(m, p)} a_{k} w_{k}(x) \leqslant 0
$$

then the Dini derivates of $f$ satisfy $D_{-} f(x) \leqslant 0 \leqslant D^{+} f(x)$.

Theorem 1 follows easily from Theorem 3 . Indeed, by considering $-f$ if necessary, we may suppose that (11) holds for all $x \in[a, b] \sim Z$. Also, since $f$ is continuous, (10) holds uniformly for $t \in[0,1]$ when $a_{k}=a_{k}(f)$ (Walsh [7]). Consequently, $D^{+} f(x) \geqslant 0 \geqslant D_{-} f(x)$ holds for all dyadic irrationals $x \in[a, b] \sim Z$, i.e., for all but countably many $x \in[a, b]$. Applying a classical theorem of Dini (see [5, p. 204]), the function $f$ is constant on $[a, b]$, as required.

To prove Theorem 3 , begin by recalling that if $x, y \in[0,1]$ have binary expansions

$$
x=\sum_{j=0}^{\infty} x_{j} 2^{-j-1}, \quad y=\sum_{j=0}^{\infty} y_{j} 2^{-j-1},
$$

then the dyadic sum of $x$ and $y$ is given by

$$
x+y=\sum_{j=0}^{\infty}\left|x_{j}-y_{j}\right| 2^{-j-1},
$$

and that

$$
w_{k}(x+y)=w_{k}(x) w_{k}(y)
$$

for $x, y \in[0,1]$ and $k=0,1, \ldots$ (Fine [3]). Thus $x+2^{-p-1}=x+2^{-p-1}$ (respectively, $x-2^{-p-1}$ ) when $x_{p}=0$ (respectively, $x_{p}=1$ ). Moreover, given a dyadic irrational $x$, there exist integers $p_{1}<p_{2}<\cdots$ and $q_{1}<q_{2}<\cdots$ such that $x_{p_{j}}=0$ 
and $x_{q_{1}}=1$ for $j \leqslant 1$. It follows that if

$$
\lim _{p \rightarrow \infty} \frac{f\left(x+2^{-p-1}\right)-f(x)}{2^{-p-1}} \geqslant 0
$$

then $\lim _{j \rightarrow \infty}\left[f\left(x+h_{j}\right)-f(x)\right] / h_{j}$ is nonnegative if $h_{j}=2^{p_{j}+1}$ and nonpositive if $h_{j}=-2^{q_{j}+1}$, i.e., $D^{+} f(x) \geqslant 0 \geqslant D_{-} f(x)$. The proof of Theorem 3 will be complete, then, if inequality (13) is verified.

Toward this, apply (10) to write

$$
f\left(x+2^{-p-1}\right)=\lim _{n \rightarrow \infty} \sum_{k=0}^{2^{n}-1} a_{k} w_{k}\left(x+2^{-p-1}\right)
$$

for $p$ sufficiently large. Hence by (10) and (12) conclude that

$$
f\left(x+2^{-p-1}\right)-f(x)=\lim _{n \rightarrow \infty} \sum_{k=0}^{2^{n}-1} a_{k} w_{k}(x)\left[w_{k}\left(2^{-p-1}\right)-1\right]
$$

holds for $p$ large. Observe by (1) that the factor $\left[w_{k}\left(2^{-p-1}\right)-1\right]$ equals 0 or -2 and that the latter case eventuates only when $k_{p}=1$, i.e., when $k \in A(m, p)$ for some $m \geqslant p$. It follows that

$$
f\left(x+2^{-p-1}\right)-f(x)=(-2) \sum_{m=p}^{\infty} \sum_{k \in A(m, p)} a_{k} w_{k}(x) .
$$

In particular, hypothesis (11) implies inequality (13) and the proof of Theorem 4 is complete.

4. A proof of Theorem 2. Set $a_{k-1}=a_{k-1}(f)$ and $D_{k}=\sum_{j=0}^{k-1} w_{k}$ for $k=1,2, \ldots$. Fix integers $m>p$ and $l \in\left[1,2^{m-p-1}\right]$ and to simplify notation, set $L=2^{m}+$ $(2 l-1) 2^{p}$ and $M=2^{m}+2 l \cdot 2^{p}-1$. Use Abel's transformation to write

$$
\sum_{k=L}^{M} a_{k} w_{k}=\sum_{k=L}^{M-1}\left(a_{k}-a_{k+1}\right) D_{k+1}+a_{M} D_{M+1}-a_{L} D_{L} .
$$

We claim that $D_{N}(x)=0$ for $2^{-p} \leqslant x<1$ and $N=M+1, L$. To verify this observe that $N=2^{\nu_{1}}+\cdots+2^{\nu_{\lambda}}$ for certain integers $\nu_{1}>\nu_{2}>\cdots>\nu_{\lambda} \geqslant p$. We take the case $\lambda=2$ as typical. According to an identity of Fine [3],

$$
D_{2^{\nu_{1}}+2^{\nu_{2}}}(x)=D_{2^{\nu_{1}}}(x)+w_{2^{\nu_{1}}}(x) D_{2^{\nu_{2}}}(x) \text {. }
$$

Moreover, $D_{2^{\nu}}(x)=0$ for $2^{-\nu} \leqslant x<1$. Since $\nu_{1}>\nu_{2} \geqslant p$ it follows that both $D_{2^{\nu_{1}}}(x)$ and $D_{2^{\nu_{2}}}(x)=0$ for $2^{-p} \leqslant x<1$.

Fix $x \neq 0$ and choose $p$ so large that $2^{-p} \leqslant x<1$. Use (14) and the fact that $D_{N}(x)=0$ to conclude that

$$
\sum_{k=L}^{M} a_{k} w_{k}(x)=\sum_{k=L}^{M-1}\left(a_{k}-a_{k+1}\right) D_{k+1}(x) .
$$

Sum these identities over all integers $l \in\left[1,2^{m-p-1}\right]$ and over $m=p, p+1, \ldots$ to conclude that

$$
\sum_{m=p}^{\infty} \sum_{k \in A(m, p)} a_{k} w_{k}(x)=\sum_{m=p}^{\infty} \sum_{k \in B(m, p)}\left(a_{k}-a_{k+1}\right) D_{k+1}(x) .
$$


Finally, use the inequality $\left|D_{k+1}(x)\right| \leqslant 2 / x$ (see [3]) to obtain

$$
\left|\sum_{m=p} \sum_{k \in A(m, p)} a_{k} w_{k}(x)\right| \leqslant\left(\frac{2}{x}\right) \sum_{m=p}^{\infty} \sum_{k \in B(m, p)}\left|a_{k}-a_{k+1}\right| .
$$

Thus (7) implies that the limit in (6) is zero, for any $x \in(0,1)$. Consequently, by Theorem 1 the function $f$ is constant as required.

\section{BIBLIOGRAPHY}

1. S. V. Bočkarev, On the Fourier-Walsh coefficients, Math. USSR-Izv. 4 (1970), 209-214.

2. J. E. Coury, Walsh series with coefficients tending monotonically to zero, Pacific J. Math. 54 (1974), $1-16$.

3. N. J. Fine, On the Walsh functions, Trans. Amer. Math. Soc. 65 (1949), 372-414.

4. C. H. Powell and W. R. Wade, Term by term dyadic differentiation, Canad. J. Math. 33 (1981), 247-256.

5. S. Saks, Theory of the integral, Hafner, New York, 1937.

6. V. A. Skvorcov and W. R. Wade, Generalizations of some results concerning Walsh series and the dyadic derivative, Anal. Math. 5 (1979), 249-255.

7. J. L. Walsh, A closed set of normal orthogonal functions, Amer. J. Math. 55 (1923), 5-24.

Department of Mathematics, University of Tennessee, Knoxville, Tennessee 37916 